\title{
ON SOLUTION OF THE INTEGRAL EQUATIONS FOR THE POTENTIAL PROBLEMS OF TWO CIRCULAR-STRIPS
}

\author{
C. SAMPATH and D.L. JAIN \\ Department of Mathematics \\ University of Delhi \\ De1hi-110007, India
}

(Received May 28, 1986 and in revised form June 20, 1988)

ABSTRACT. Solutions are given to some singular integral equations which arise in twodimensional Dirichlet and Newmann boundary value problems of two equal infinite coaxial circular strips in various branches of potential theory. For illustration, these solutions are applied to solve some boundary value problems in electrostatics, hydrodynamics, and expressions for the physical quantities of interest are derived.

KEY WORDS AND PHRASES. Infinite co-axial circular strips, Chebychev polynomials, surface charge density, kinetic energy, elliptic integrals of the first and the second kinds.

1980 AMS SUBJECT CLASSIFICATION CODE. 31 .

\section{INTRODUCTION.}

Recently many authors [1-5] have presented solutions of various two dimensional boundary value problems of two infinite strips, by integral equation techniques [68]. Shail [9] has given the solution of the Fredholm singular integral equation of the first kind with logarithmic kernal $\left\{q+\log 2\left|a \sin \frac{1}{2}\left(\theta-\theta_{1}\right)\right|\right\},-\alpha<\theta_{1}, \theta<\alpha$, where $a$ and $q$ are known constants. This type of singular integral equation governs the solutions of various two-dimensional Dirichlet boundary value problems involving an infinite circular strip in electrostatics, hydrodynamics, and low-frequency acoustic scattering. The solution to this singular integral equation is derived in a closed form from those of some well known integral equations of Carlemann type [10, $11,12]$.

We present here solutions of Fredholm singular integral equations of the first kind of the type

$$
\int_{-\alpha}^{-\beta}+\int_{\beta}^{\alpha} g\left(\theta_{1}\right)\left\{q+\log \left|2 a \sin \frac{1}{2}\left(\theta-\theta_{1}\right)\right|\right\} d \theta_{1}=f(\theta), \beta<|\theta|<\alpha,
$$


where the known function $f(\theta)$ is of class $c^{1}$, for the values of $\theta$ satisfying the inequality $0<\beta<|\theta|<\alpha \pi$, and a $q$ are known constants. The singular integral equations of the type (1.1) govern solutions of various two-dimensional Dirichlet boundary value problems of two equal infinite co-axial circular strips in potential theory. In the corresponding Newmann boundary value problems, the governing integral equations are of the type

$$
\int_{-\alpha}^{-\beta}+\int_{\beta}^{\alpha} I\left(\theta_{1}\right) \operatorname{cosec}^{2} \frac{1}{2}\left(\theta-\theta_{1}\right) d \theta_{1}=f(\theta), \beta<|\theta|<\alpha,
$$

where the unkown density function satisfies the edge condtions

$$
I( \pm \beta)=I( \pm \alpha)=0
$$

Integrating by parts and using edge conditions (1.3), (1.2) becomes

$$
\int_{-\alpha}^{-\beta}+\int_{\beta}^{\alpha} g\left(\theta_{1}\right) \cot \frac{1}{2}\left(\theta-\theta_{1}\right) d \theta_{1}=\frac{1}{2} f(\theta), \quad \beta<|\theta|<\alpha
$$

where $g(\theta)=I^{\prime}(\theta)$.

We present here a simple technique of solving integral equations of the types (1.1) and (1.2). For illustration, this technique is applied to solve an electrostatic Dirichlet boundary value problem and a hydrodynamic Newmann boundary value problem of two equal infinite co-axial circular strips.

We have also solved the two-dimensional problems of scattering of a low-frequency incident plane acoustic ware by two equal infinite co-axial soft and rigid circular strips by the integral equation technique. This work will appear separately.

The plan of this paper is as follows. In section 2, we first present a simple technique of solving integral equations of the type (1.1) without reducing it to some well known integral equations of the Carleman type $[10,11,12]$. This is achieved by reducing the solution of (1.1) to that of two Fredholm singular integral equations of the first kind with kernels (i) (Const.) $+\log |2(\cos x-\cos y)|, 0<x, y<\pi$, and (ii) $(\cos y-\cos x)^{-1}, 0<x, y<\pi$. The unknown and known functions are both even degree functions in each of these two integral equations. The first of these two equations readily yields the Fourier expansion of the unknown even degree funtion over the interval $0<y<\pi$, when the well known expansion of the the kerne1 [1,7] and the Fourier expansion of the known even degree function over the interval $0<x<\pi$ are made in it. Similarly, we obtain the series expansion of the unknown even degree function of the second integral equation in terms of Chebychev polynomials $T_{n}$ (cosy) of the first kind when we use the series expansion of its known even degree function in terms of Chebychev polynomials $U_{n}(\cos x)$ of the second kind. The solution of this second integral equation contains an unknown constant which is evaluated by making its solution to satisfy an appropriate inner edge condition. Then we illustrate this 
technique to solve the integral equation (1.1), when the known function $f(\theta)$ in it is of a particular form, for our subsequent analysis. Lastly, we explain how equation (1.2) can be transformed to the form of integral equation (1.1) and is therefore solvable by the above technique.

In Section 2 we apply the integral equation technique given in Section 1 to solve the two-dimensional electrostatic Dirichlet boundary value problem of two equal infinite co-axial perfectly conducting clrcular strips in a free space, when the total charge per unit height of the two strips is unity. Section 3 is devoted to the study of the two-dimensional hydrodynamic Newmann boundary value problem of uniform flow of an inviscid homogeneous liquid streaming past two equal infinite co-axial fixed rigid circular strips. The expression for the kinetic energy per unit height of the secondary fluid flow is derived.

2. SOLUTIONS OF INTEGRAL EQUATIONS.

We present here a simple technique to derive solutions of equations (1.1) and $(1.2)$.

INTEGRAL EQUATION (1.1): To solve equation (1.1) we first substitute in it [11]

$$
f(\theta)=f_{1}(\theta)+f_{2}(\theta) ; g(\theta)=g_{1}(\theta)+g_{2}(\theta)
$$

where the subscripts 1 and 2 represent the even degree and the odd degree parts of the corresponding functions respectively. These substitutions readily decouple the integral equation (1.1) into the following two integral equations

$$
\begin{aligned}
& \int_{\beta}^{\alpha} g_{1}\left(\theta_{1}\right)\left\{2 q+\log \left|2 a^{2}\left(\cos \theta-\cos \theta_{1}\right)\right|\right\} d \theta_{1}=f_{1}(\theta), \beta<\theta<\alpha, \\
& \int_{\beta}^{\alpha} g_{2}\left(\theta_{1}\right) \log \left|\frac{\sin \frac{1}{2}\left(\theta-\theta_{1}\right)}{\sin \frac{1}{2}\left(\theta+\theta_{1}\right)}\right| \mathrm{d} \theta_{1}=f_{2}(\theta), \quad \beta<\theta<\alpha,
\end{aligned}
$$

for the determination of $g_{1}(\theta)$ and $g_{2}(\theta)$. We first solve equation (2.2). The substitutions

$$
\begin{aligned}
& \cos \theta_{1}=\frac{1}{2}\{(\cos \beta-\cos \alpha) \cos y+(\cos \beta+\cos \alpha)\}, \\
& \cos \theta=\frac{1}{2}\{(\cos \beta-\cos \alpha) \cos x+(\cos \beta+\cos \alpha)\},
\end{aligned}
$$

reduce the equation $(2.2)$ to a simple form

$$
\int_{0}^{\pi} G_{1}(y)\{\Delta+\log 2|\cos x-\cos y|\} d y=F_{1}(x), \quad 0<x<\pi,
$$

where

$$
\begin{aligned}
& G_{1}(y)=\left[\frac{1}{2}(\cos \beta-\cos \alpha) g_{1}\left(\theta_{1}\right) \sinh \right] / \sin \theta_{1}=\left[g_{1}\left(\theta_{1}\right) R\left(\theta_{1}\right)\right] / \sin \theta_{1}, \\
& R(\theta)=[\cos \beta-\cos \theta)(\cos \theta-\cos \alpha)]^{1 / 2},
\end{aligned}
$$




$$
F_{1}(x)=f_{1}(\theta), \Delta=2 q+\log \left[a^{2}(\cos \beta-\cos \alpha) / 2\right],
$$

and in (2.7) we have used the relations

$$
\sin \frac{y}{2}=\left(\frac{\cos \beta-\cos \theta}{\cos \beta-\cos \alpha}\right)^{1 / 2}, \cos \frac{y}{2}=\left(\frac{\cos \theta_{1}-\cos \alpha}{\cos \beta-\cos \alpha}\right)^{1 / 2},
$$

readily derivable from the substitution (2.4). Lastly, the well known formula [7]

$$
\log 2|\cos y-\cos x|=-2 \sum_{n=1}^{\infty} \frac{\cos n x \cos n y}{n}, \quad 0<x, y<\pi
$$

and the Fourier expansion of the known even degree function $F_{1}(x)$

$$
F_{1}(x)=\frac{1}{2} a_{0}+\sum_{n=1}^{\infty} \underset{n}{a} \cos n x, \quad 0<x<\pi,
$$

where $\quad a_{n}=\frac{2}{\pi} \int_{0}^{\pi} F_{1}(\cos n x d x, n>0$,

readily yield the solution $G_{1}(y)$ of the equation (2.6). This is given by

$$
G_{1}(y)=b_{0}+\sum_{n=1}^{\infty} b_{n} \cos n y,
$$

where $\quad b_{0}=a_{0} /(2 \pi \Delta), b_{n}=-\left(n a_{n}\right) / \pi, n>1$,

and the known coefficients $a_{n}, n>0$, are defined by the relations (2.13). Finally, substituting the above value of $G_{1}(y)$ in the relation (2.7), we obtain the required solution of the equation (2.2)

$$
g_{1}\left(\theta_{1}\right)=\left[b_{0}+\sum_{n=1}^{\infty} b_{n} \cos n y\right]\left[\sin \theta_{1}\right] / R\left(\theta_{1}\right), \quad \beta<\theta_{1}<\alpha,
$$

Now we take up the solution of the equation (2.3). We first differentiate both sides of equation (2.3) with respect to $\theta$ and obtain

$$
\int_{\beta}^{\alpha} \frac{g_{2}\left(\theta_{1}\right) \sin \theta_{1}}{\cos \theta_{1}-\cos \theta} d \theta_{1}=f_{2}^{\prime}(\theta), \quad \beta<\theta<\alpha,
$$

where the integral in the left hand member is to be inerpreted as a Cauchy principal value. When we make the substitutions $(2.4)$ and $(2.5)$ in the above equations, we get

$$
\int_{0}^{\pi} \frac{G_{2}(y)}{\cos y-\cos x} d y=F_{2}(x), \quad 0<x<\pi,
$$

where

$$
\begin{aligned}
& G_{2}(y)-g_{2}\left(\theta_{1}\right) \text { siny }=\left[2 g_{2}\left(\theta_{1}\right) R\left(\theta_{1}\right)\right] /[\cos \beta-\cos \alpha], \\
& F_{2}(x)=f_{2}^{\prime}(\theta),
\end{aligned}
$$

and $F_{2}(x)$ is a known even degree function of $x$. Next, when we substitute 


$$
x=\cos ^{-1}(X), y=\cos ^{-1}(Y),
$$

in (2.18), we obtain

$$
\int_{-1}^{1} \frac{G_{2}\left(\cos ^{-1} Y\right) d Y}{\left(1-Y^{2}\right)^{1 / 2(Y-X)}} \quad F_{2}\left(\cos ^{-1} X\right),-1<X<1 .
$$

The above integral equation can be readily solved when we substitute it in the expansion of the known function $F_{2}$ in terms of the Chebychev polynomials $U_{n}(X)$ of the second kind

$$
F_{2}\left(\cos ^{-1} X\right)=\sum_{n=1}^{\infty} c_{n} U_{n}(X), \quad-1<x<1,
$$

and use the well known formula

$$
\int_{-1}^{1} \frac{T_{n}(Y) d Y}{\left(1-Y^{2}\right)^{1 / 2}(Y-X)}= \begin{cases}0 & n=0, \\ \pi U_{n-1}(X), & n>1,\end{cases}
$$

where $T_{n}(Y)$ is the $n t h$ degree Chebychev polynomial of the first kind. Thus the solution of the equation (2.22) is given by

$$
G_{2}(y)=A T_{0}(\cos y)+\frac{1}{\pi} \sum_{n=1}^{\infty} c_{n-1} T_{n}(\cos y), \quad 0<y<\pi,
$$

where $A$ is an arbitrary constant and the constant coefficients $c_{n}$ are defined by the expansion (2.23) of the known function $\mathrm{F}_{2}$. These values are

$$
c_{n}=\frac{2}{\pi} \int_{0}^{\pi} \sin x \sin (n+1) x F_{2}(x) d x, \quad n>0 .
$$

Lastly, relations (2.19) and (2.25) lead to the required solution of the equation $(2.3)$

$$
g_{2}\left(\theta_{1}\right)=\frac{1}{2}(\cos \beta-\cos \alpha)\left[A+\frac{1}{\pi} \sum_{n=1}^{\infty} c_{n-1} \cos n y\right] / R\left(\theta_{1}\right), \quad \beta<\theta_{1}<\alpha,
$$

where relation (2.4) gives the value of cosy in terms of $\cos \theta_{1}$. The value of the constant A in (2.27) is readily obtained by using the edge condition satisfied by the density function $g_{2}\left(\theta_{1}\right)$ at the inner edge $\theta_{1}=\beta$. This edge condition is

$$
g_{2}\left(\theta_{1}\right)=0\left(\left[\cos \beta-\cos \theta_{1}\right]^{1 / 2}\right) \text {, as } \theta_{1} \rightarrow \beta+0
$$

The value of $g_{2}\left(\theta_{1}\right)$ given by (2.27) satisfies this edge condition if in (2.25), $G_{2}(y) \rightarrow 0$, as $y \rightarrow 0+$, and hence

Therefore,

$$
A=-\frac{1}{\pi} \sum_{n=1}^{\infty} c_{n-1}
$$

$$
g_{2}\left(\theta_{1}\right)=\frac{-1}{2 \pi}(\cos \beta-\cos \alpha)\left[\sum_{n=1}^{\infty} c_{n-1}(1-\cos n y)\right] / R\left(\theta_{1}\right), \quad \beta<\theta_{1}<\alpha .
$$


Since, $\sin ^{2} \frac{1}{2} y$ or $\left(\frac{\cos \beta-\cos \theta}{\cos \beta-\cos \alpha}\right)$ is a common factor of all the terms occuring in the infinite series in the right member of the above equation, therefore, the above expression for $\mathrm{g}_{2}\left(\theta_{1}\right)$ satisfies the required edge condition (2.28). Finally, relations $(2.1),(2.16)$ and $(2.30)$ yield the required solution $g\left(\theta_{1}\right)$ of equation (1.1). We illustrate the above results by solving the integral equation, $\alpha<|\theta|<\beta$,

$\int_{-\alpha}^{-\beta}+\int_{\beta}^{\alpha} g\left(\theta_{1}\right)\left\{q+\log \left|2 a \sin \frac{1}{2}\left(\theta-\theta_{1}\right)\right|\right\} d \theta_{1}=\left[B_{10}+B_{11} \cos \theta\right]+\left[B_{20} \theta+B_{21} \sin \theta\right],(2.31)$ where all B's are known constants. Its solution is also very useful in solving various boundary value problems presented in our subsequent analysis. Comparing equation (2.31) with (1.1), we have, in this case,

$$
\begin{aligned}
& f_{1}(\theta)=B_{10}+B_{11} \cos \theta, f_{2}(\theta)=B_{20} \theta+B_{21} \sin \theta \\
& F_{1}(x)=\left[B_{i 0}+\frac{1}{2} B_{i 1}(\cos \beta+\cos \alpha)\right]+\frac{1}{2} B_{11}(\cos \beta-\cos \alpha) \cos x, i=1,2, \\
& G_{1}(y)=\frac{1}{\pi}\left\{\frac{1}{\Delta}\left[B_{10}+\frac{1}{2} B_{11}(\cos \beta+\cos \alpha)\right]-\frac{1}{2} B_{11}(\cos \beta-\cos \alpha) \cos y\right\} \\
& G_{2}(y)=\frac{-1}{\pi}\left\{\left[B_{20}+\frac{1}{2} B_{21}(\cos \beta+\cos \alpha)\right](1-\cos y)+\frac{1}{4}\left[B_{21}(\cos \beta-\cos \alpha)\right](1-\cos 2 y)\right\}(2.35) \\
& g_{1}\left(\theta_{1}\right)=\frac{1}{\pi}\left(\sin \theta_{1}\right)\left\{\frac{1}{\Delta} B_{10}+\frac{1}{2}\left(\frac{1}{\Delta}+1\right) B_{11}(\cos \beta+\cos \alpha)\right\} \\
& \left.\left.g_{2}\left(\theta_{1}\right)=\frac{-1}{\pi}\left(\cos \beta-\cos \theta_{1}\right) B_{11} \cos \theta_{1}\right\} / R\left(\theta_{1}\right), B<\theta_{1}<\alpha, 33\right) \\
& \left.\quad+B_{20}+\frac{1}{2} B_{21}(\cos \beta-\cos \alpha)\right]
\end{aligned}
$$

and putting the values of the functions $g_{1}, g_{2}$ from equations (2.36) and (2.37) in the relation $g\left(\theta_{1}\right)=g_{1}\left(\theta_{1}\right)+g_{2}\left(\theta_{2}\right)$, we get the required solution of equation $(2.31)$. In the above illustration, when $\beta \rightarrow 0$, we readily obtain the solution of the equation, $-\alpha<\theta<\alpha$,

$$
\int_{-\alpha}^{\alpha} g\left(\theta_{1}\right)\left\{q+\log \mid 2 a \sin \frac{1}{2}\left(\theta-\theta_{1}\right) d \theta=\left(B_{10}+B_{11} \cos \theta\right)+\left(B_{20} \theta+B_{21} \sin \theta\right),\right.
$$

in the $\mathrm{f}$ orm

$$
\begin{aligned}
& g\left(\theta_{1}\right)=\frac{1}{\pi} \sqrt{2}\left[\cos \frac{1}{2} \theta_{1}\left\{\frac{1}{\Delta_{1}} B_{10}+B_{11}\left[\left(\frac{1}{\Delta_{1}}+1\right) \cos ^{2} \frac{1}{2} \alpha-\cos \theta_{1}\right]\right\}\right. \\
& \left.-\sin \frac{1}{2} \theta_{1}\left\{B_{20}+B_{21}\left[\sin ^{2} \frac{1}{2} \alpha+\cos \theta_{1}\right]\right\}\right] /\left(\cos \theta_{1}-\cos \alpha\right)^{1 / 2},-\alpha<\theta_{1}<\alpha,
\end{aligned}
$$

where

$$
\Delta_{1}=2\left[q+\log \left(a \sin \frac{1}{2} \alpha\right)\right],
$$


which agrees with results derived by Shall [9] by applying tedious inversion formulas. INTEGRAL EQUATION (1.2). We present here the method of solving equation (1.2) or its equivalent form (1.4). Equation (1.4) can be rewritten in the form

$$
\frac{d}{d \theta} \int_{-\alpha}^{-\beta}+\int_{\beta}^{\alpha} g\left(\theta_{1}\right) \log \left|\sin \frac{1}{2}\left(\theta-\theta_{1}\right)\right| d \theta_{1}=-\frac{1}{4} f(\theta), \beta<|\theta|<\alpha,
$$

which on integration of the both nembers of this equation yields

$$
\int_{-\alpha}^{-\beta}+\int_{\beta}^{\alpha} g\left(\theta_{1}\right) \log \left|\sin \frac{1}{2}\left(\theta-\theta_{1}\right)\right| \mathrm{d} \theta_{1}=c-\frac{1}{4} \mathrm{p}(\theta), \infty<|\theta|<\alpha,
$$

where $\mathrm{p}^{\prime}(\theta)=\mathrm{f}(\theta)$ and $C$ in an unknown constant. Since the above inegral equation is also of the form $(1.1)$, with $f(\theta)=c-\frac{1}{4} p(\theta), q=-\log 2$, and $a=1$, therefore, its solution $g\left(\theta_{1}\right)$ can be derived as explained in section 2.1. Lastly, the value of the unknown constant $C$ occuring in the even degree part $g_{1}\left(\theta_{1}\right)$ of this solution

$g\left(\theta_{1}\right)=g_{1}\left(\theta_{1}\right)+g_{2}\left(\theta_{1}\right)$, can be obtained by putting this value of $g_{1}\left(\theta_{1}\right)$ in the relation

$$
\int_{\beta}^{\alpha} g_{1}\left(\theta_{1}\right) d \theta_{1}=\frac{1}{2} \int_{-\alpha}^{-\beta}+\int_{\beta}^{\alpha} g\left(\theta_{1}\right) d \theta_{1}=\frac{1}{2} \int_{-a}^{-\beta}+\int_{\beta}^{\alpha} I^{\prime}\left(\theta_{1}\right) d \theta_{1}=0,
$$

where we have used relation (1.5) and the edge condition (1.3)).

\section{ELECTROSTATIC POTENTIAL PROBLEM.}

We consider the electrostatic problem of two equal infinite co-axial perfectly conducting strips charged in a free space so that the total charge per unit height on the two strips is unity. In cylindrical polar co-ordinates $(r, \theta, z)$, the two strips are defined by $\mathbf{r}=\mathrm{a},-\alpha,\langle\theta<-\beta, \beta<\theta<\alpha, \beta>0, a<\pi,-\infty<z<\infty$.

The electrostatic potential $\phi(r, \theta)$ of this boundary value problem is given by

$$
\phi(r, \theta)=-a \int_{-\alpha}^{-\beta}+\int_{\beta}^{\alpha} g\left(\theta_{1}\right) \log \left\{r^{2}+a^{2}-2 \operatorname{arcos}\left(\theta-\theta_{1}\right)\right\}^{1 / 2} d \theta_{1} \text {, }
$$

where $g\left(\theta_{1}\right)$ is the unknown surface charge density per unit are defined by

$$
g\left(\theta_{1}\right)=-\frac{1}{2 \pi}\left[\frac{\partial \phi}{\partial r}\left(r, \theta_{1}\right)\right]_{r=a^{-}}^{r=a+}, \beta<\left|\theta_{1}\right|<\alpha,
$$

Since the value of the potential $\phi$ assumes a constant value, say $\phi_{0}$, on the two strips, using this boundary condition in ( 3.1$)$, we obtain the integral equation

$$
\int_{-\alpha}^{-\beta}+\int_{\beta}^{\alpha} g\left(\theta_{1}\right) \log \left|2 a \sin \frac{1}{2}\left(\theta-\theta_{1}\right)\right| d \theta_{1}=\frac{\phi_{0}}{a}, \quad \beta<|\theta|<\alpha,
$$


The solution of this equation is readily obtained from that of equation (2.31), by setting $q=0, B_{10}=-\phi_{0} / a, B_{11}=B_{20}=B_{21}=0$, in (2.36) and (2.37). Therefore, the solution of equation ( 3.1 ) is given by

$$
g\left(\theta_{1}\right)=g_{1}\left(\theta_{1}\right)=-\frac{1}{\pi}\left[\phi_{0} /\left(a \Delta_{2}\right)\right]\left|\sin \theta_{1}\right| / R\left(\theta_{1}\right), \quad \beta<\left|\theta_{1}\right|<\alpha,
$$

where $\quad \Delta_{2}=\log \left[a^{2}(\cos \beta-\cos \alpha) / 2\right]$.

Finally, to evaluate the unknown constant $\phi_{0}$ in the above expression for the charge density $g\left(\theta_{1}\right)$ of the strips, it is given that the total charge per unit height on each of the strip is unity and therefore $g\left(\theta_{1}\right)$ must satisfy the condition

$$
a \int_{-\alpha}^{-\beta}+\int_{\beta}^{\alpha} g\left(\theta_{1}\right) d \theta_{1}=2
$$

We substitute the value of $g\left(\theta_{1}\right)$ from (3.4) in the above equation, and get

$$
\phi_{0}=-\Delta_{2}
$$

and, therefore,

$$
g\left(\theta_{1}\right)=\frac{1}{\pi a}\left|\sin \theta_{1}\right| / R\left(\theta_{1}\right), \quad B<\left|\theta_{1}\right|<\alpha,
$$

When $B+0$, we obtain the corresponding limiting result [9] for the circular strip $\mathbf{r}=a,-\alpha<\theta<\alpha,-\infty<z<\infty$

$$
g\left(\theta_{1}\right)=\frac{\sqrt{2} \cos \frac{1}{2} \theta_{1}}{\pi a\left(\cos \theta_{1}-\cos \alpha\right)^{1 / 2}},-\alpha<\theta_{1}<\alpha .
$$

\section{HYDRODYNAMICAL PROBLEM.}

We consider the problem of uniform flow of an inviscid homogeneous liquid in the direction $\hat{n}=\hat{i} \cos \gamma+\hat{j} s i n \gamma$ of velocity $U$, streaming past two fixed rigid strips $r=$ a, $-\alpha<\theta-\beta, \beta<\theta<\alpha, 0<\beta<\alpha<\pi$. The secondary velocity potential function $\phi_{s}(r, \theta)$ of this two-dimensional problem is given by [9]

$$
\phi_{s}(r, \theta)=-\frac{a}{2 \pi} \int_{-\alpha}^{-\beta}+\int_{\beta}^{\alpha} I\left(\theta_{1}\right)\left\{\frac{\partial}{\partial r_{1}} \log \left[r^{2}+r_{1}^{2}-2 r r_{1} \cos \left(\theta-\theta_{1}\right)\right]^{1 / 2}\right\} d \theta_{1}, \quad r_{1}=a
$$

where

$$
I\left(\theta_{1}\right)=\phi_{s}\left(a+, \theta_{1}\right)-\phi_{s}\left(a-, \theta_{1}\right), \beta<\left|\theta_{1}\right|<\alpha,
$$

and $I\left(\theta_{1}\right)$ satisfies the edge condition

$$
I( \pm \beta)=I( \pm \alpha)=0
$$


Using the boundary condition

$$
\left[\frac{\partial \phi}{\partial r}\right]_{r=a}=-\left[\frac{\partial \phi}{\partial r}\right]_{r=a}
$$

where $\dot{\phi}_{i}(r, \theta)=-U r \cos (\theta-\gamma)$ is the velocity potential function of the incident uniforn flow Un, we obtain from (4.1), the integral equation [9]

$$
\int_{-\alpha}^{-\beta}+\int_{\beta}^{\alpha} I\left(\theta_{1}\right) \operatorname{cosec}^{2} \frac{1}{2}\left(\theta-\theta_{1}\right) \mathrm{d} \theta_{1}=8 \pi \operatorname{aUcos}(\theta-\gamma), \beta<|\theta|<\alpha,
$$

To solve this integral equation, we first integrate the left member by parts and obtain by using the edge conditions (4.3)

$$
\int_{-\alpha}^{-\beta}+\int_{\beta}^{\alpha} g\left(\theta_{1}\right) \cot \frac{1}{2}\left(\theta-\theta_{1}\right) \mathrm{d} \theta_{1}=-4 \pi \mathrm{aU} \cos (\theta-\gamma), \beta<|\theta|<\alpha,
$$

where $g(\theta)=I^{\prime}(\theta)$. The above integral equation is of the type (1.4), and therefore can be solved by the method given in the section 2.2. We first rewrite integral equation (4.6) in the form (2.41) which on integration yields

$$
\int_{-\alpha}^{-\beta}+\int_{\beta}^{\alpha} g\left(\theta_{1}\right) \log \left|\sin \frac{1}{2}\left(\theta-\theta_{1}\right)\right| d \theta_{1}=C-2 \pi \operatorname{aUsin}(\theta-\gamma), \beta<|\theta|<\alpha,(4.7)
$$

where $C$ is an unknown constant. Fortunately, the aboe integral equation is of the type (2.31), with $q=10 g 2, a=1, B_{10}=C, B_{11}=2 \pi a \sin \gamma, B_{20}=0, B_{21}=-2 \pi a U \cos \gamma$. Therefore, substituting these values of the constants in equations (2.36) and (2.37), we obtain the required solution of the above integral equation in the form

$$
g\left(\theta_{1}\right)=g_{1}\left(\theta_{1}\right)+g_{2}\left(\theta_{1}\right)
$$

where

$$
\begin{aligned}
& g_{1}\left(\theta_{1}\right)=\frac{1}{\pi}\left|\sin \theta_{1}\right|\left\{\frac{1}{\delta} C+\pi \operatorname{aUsin\gamma }\left[(\cos \beta+\cos \alpha)\left(\frac{1}{\delta}+1\right)-2 \cos \theta_{1}\right]\right\} / R\left(\theta_{1}\right), \beta<|\theta|<\alpha,(4.9) \\
& \text { and } \quad \delta=\log \{(\cos \beta-\cos \alpha) / 8\}
\end{aligned}
$$

The value of the unknown constant $C$ occuring in the value of $g_{1}\left(\theta_{1}\right)$ given by equation (4.9) is obtained by putting this value of $g_{1}\left(\theta_{1}\right)$ in the relation (2.43). This yields the required value of the unknown constant $C$ is given by

$$
C=-\pi a U \sin \gamma(\cos \beta+\cos \alpha)
$$

We substitute this value of $C$ in $(4.9)$ to obtain the value of the function $g_{1}\left(\theta_{1}\right)$ as

$$
\mathrm{g}_{1}\left(\theta_{1}\right)=\mathrm{aU} \sin \gamma\left|\sin \theta_{1}\right|\left[(\cos \beta+\cos \alpha)-2 \cos \theta_{1}\right] / R\left(\theta_{1}\right), \beta<|\theta|<\alpha
$$


Using formulas (2.19) and (2.25), the expression for $g_{2}\left(\theta_{1}\right)$ is given by

$$
g_{2}\left(\theta_{1}\right)=\frac{A}{R\left(\theta_{1}\right)}\left[C-2 a U \cos \gamma\left(B \cos y+\frac{A}{2} \cos 2 y\right)\right], B<|\theta|<\alpha
$$

where

$$
A=\frac{1}{2}(\cos \beta-\cos \alpha), B=\frac{1}{2}(\cos \beta+\cos \alpha), \cos \theta_{1}=A \cos y+B,
$$

and the unknown constant $C$ is evaluated by using the condition (1.3) which yields

$$
C=2 \mathrm{aU} \cos \gamma\left[\mathrm{B} J+\frac{\mathrm{A}}{2} \mathrm{~J}_{2}\right] / \mathrm{J}_{\mathrm{o}}
$$

where $\quad J_{n}=\int_{\beta}^{\alpha} \frac{(\cos n y) d \theta_{1}}{R\left(\theta_{1}\right)}, n=0,1,2$,

We substitute this value of $\mathrm{C}$ in (4.13) to obtain the following value of $\mathrm{g}_{2}\left(\theta_{1}\right)$.

$$
\begin{aligned}
& g_{2}\left(\theta_{1}\right)=\frac{2 a U \cos \gamma}{R\left(\theta_{1}\right)} \operatorname{sgn} \theta_{1}\left[B\left(\frac{J_{1}}{J_{0}}-\cos y\right)+\frac{A}{2}\left(\frac{J_{2}}{J_{0}}-\cos 2 \gamma\right)\right] \\
& =\frac{2 a U \cos \gamma}{R\left(\theta_{1}\right)} \operatorname{sgn} \theta_{1}\left[\frac{A^{2}}{2}+\frac{A}{J_{0}}\left(B J_{1}+\frac{A}{2} J_{2}\right)+B \cos \theta_{1}-\cos ^{2} \theta_{1}\right], B<\left|\theta_{1}\right|<\alpha,
\end{aligned}
$$

The results $(4.8),(4.12)$ and $(4.17)$ yield the required solution $g\left(\theta_{1}\right)$ of the integral equation (4.6). Since $g(\theta)=I^{\prime}(\theta), \beta\left|\theta_{1}\right|<\alpha$, therefore

$$
I(\theta)=\operatorname{sgn} \theta \int_{\beta}^{|\theta|} g_{1}\left(\theta_{1}\right) d \theta_{1}+\int_{\beta}^{|\theta|} g_{2}\left(\theta_{1}\right) d \theta_{1}, \beta<|\theta|<\alpha,
$$

where we have used the relation (4.8). Since the expression (4.12)

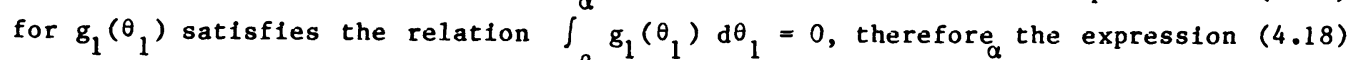
for $I(\theta)$ satisfies all the required edge conditions $(4.3)$ if $\int_{\beta}^{\beta} g_{2}\left(\theta_{1}\right) d \theta_{1}=0$.

We may remark here that there is no need of obtaining the solution $I(\theta)$ of the equation (4.5) for finding the expressions for the physical quantities of interest. These expressions can be readily derived from the value of the function $g\left(\theta_{1}\right)$ given by relations (4.8) to (4.12). For instance, the kinetic energy per unit height (K.E.) of the secondary fluid flow is given by

$$
\begin{aligned}
& \text { K.E. }=-\frac{1}{2} a \rho \int_{-\alpha}^{-\beta}+\int_{\beta}^{\alpha} I\left(\theta_{1}\right)\left[\frac{\partial \phi_{s}\left(r_{1}, \theta_{1}\right)}{\partial r_{1}}\right]_{r_{1}=a} d \theta_{1} \\
& =-\frac{U a \rho}{2} \int_{-\alpha}^{-\beta}+\int_{\beta}^{\alpha} I\left(\theta_{1}\right) \cos \left(\theta_{1}-\gamma\right) d \theta_{1} \\
& \frac{U a \rho}{2} \int_{-\alpha}^{-\beta} g\left(\theta_{1}\right) \sin \left(\theta_{1}-\gamma\right) d \theta_{1}=\operatorname{Ua\rho }\left\{d_{1} \cos \gamma-d_{2} \sin \gamma\right\},
\end{aligned}
$$

where we have used the boundary condition $(4.4)$, the relation $g(\theta)=I^{\prime}(\theta)$, the edge 
conditions (4.3), and $p$ is the density of the homogeneous liquid. The values of the constants $d_{1}$ and $d_{2}$ are given by the relations

$$
d_{1}=\int_{\beta}^{\alpha} g_{2}\left(\theta_{1}\right) \sin \theta_{1} d \theta_{1}, \quad d_{2}=\int_{\beta}^{\alpha} g_{1}\left(\theta_{1}\right) \cos \theta_{1} d \theta_{1}
$$

We substitute the values of the functions $g_{1}\left(\theta_{1}\right)$ and $g_{2}\left(\theta_{1}\right)$ from equations $(4.12)$ and $(4.17)$ in $(4.20)$ to obtain

$$
\mathrm{d}_{1}=2 \mathrm{a} \pi \mathrm{UA} \cos \gamma\left[\mathrm{BJ}_{1}+\frac{\mathrm{A}}{2} \mathrm{~J}_{2}\right] / \mathrm{J}_{0}, \mathrm{~d}_{2}=-\frac{\pi}{4} \mathrm{aU}(\cos \beta-\cos \alpha)^{2} \sin \gamma \cdot
$$

Finally, relations $(4.19)$ and $(4.21)$ give rise to the required expression for Kinetic energy and this is given by

$$
\text { K.E. } \left.=\rho a^{2} U^{2} \pi A\left\{\left[\frac{1}{J_{0}}+\frac{A}{2} J_{2}\right)+\frac{A}{2}\right]+\left[\frac{1}{J_{0}}\left(B J_{1}+\frac{A}{2} J_{2}\right)-\frac{A}{2}\right] \cos 2 \gamma\right\},
$$

where the definite integrals $J_{n}, n=0,1,2$ are defined by relation (4.16) and $A$ and $B$ are defined in the relation $(4.14)$.

We derive now some interesting limiting results from the formula (4.2) for K.E. When $B \rightarrow 0, J_{n} \rightarrow \infty, n=0,1,2$ and $\frac{J_{1}}{J_{0}}, \frac{J_{2}}{J_{0}} \rightarrow 1$ and therefore the formula (4.22) yields the following corresponding limiting expression for the $K_{0} E_{,}, \sigma_{1}$ in case of the infinite circular rigid strip $r=a,-\alpha<\theta<\alpha,-\infty<z<\infty$ :

$$
\sigma_{1}=r^{2} u^{2} \pi \sin ^{2} \frac{\alpha}{2}\left[1+\cos ^{2} \frac{\alpha}{2} \cos 2 \gamma\right],
$$

This seems to be a new result.

Similarly, when in formula (4.22) we let $\alpha \rightarrow 0, \beta \rightarrow 0, a+\infty$, such that $a \alpha \rightarrow a_{1}$ and $a \beta \rightarrow a_{2}\left(a_{2}<a_{1}\right)$, we obtain the corresponding limiting expression for the Kinetic Equation $\sigma_{2}$ in case of the two equal parallel co-planar infinite rigid strips $x=0, a_{2}<|y|<a_{1},-\infty<z<\infty$ and it is given by

$$
\sigma_{2}=\frac{\rho U^{2} a_{1}^{2} \pi}{2}\left[\left(1+c^{2}\right)-2 E^{\prime} / F^{\prime}\right] \cos ^{2} Y
$$

where

$$
c=a_{2} / a_{1}<1, F^{\prime}=F\left(\frac{\pi}{2},\left(1-c^{2}\right)^{1 / 2}\right), E^{\prime}=E\left(\frac{\pi}{2},\left(1-c^{2}\right)^{1 / 2}\right),
$$

and $F\left(\frac{\pi}{2}, c\right)$ and $E\left(\frac{\pi}{2}, c\right)$ are elliptic integrals of the first and the second kind [14].

We have also solved the two-dimensional problems of scattering of a low-frequency incident plane acoustic wave by the integral equation techniques presented here. This 
work will appear separately.

ACKNOWLEDGMENT. This research work was supported by a grant froin the University Grants Commission, New Delhi (India).

\section{REFERENCES}

1. GOEL, G.C. and JAIN, D.L. A note on electrostatic problem involving two strips, J. Pure App1. Math. 7(1976), 751-756.

2. GOEL, G.C. and JAIN, D.L. Electrostatic problems of two co-planar paralle1 strips, Indian J. Pure Appl. Math. 근 1976), 809-816.

3. JAIN, D.L. and KANWAL, R.P. Acoustic diffraction of a plane wave by two coplanar parallel perfectly soft or rigid strips,Can. J. Phys. 50(1972), 929939.

4. JAIN, D.L. and KANWAL, R.P. Diffraction of elastic waves by two co-planar and parallel rigid strips, Int. J. Engg. Sci. 10(1972), 925-937.

5. KANWAL R.P. and SACHDEVA, B.K. Approximate solutions of certain integral equations for the diffraction of two strips, ZAMP, 24(1973), 111-119.

6. GAUTESEN, A.K. and OLMSTEAD, W.E. On the solution of the integral equation for the potential of two strips, SIAM J. Math. Ana1., 2(1971), 293-306.

7. MILlaR, R.F. A note on diffraction by an infinite slit, Can. J. Phys., $38(1960), 38-47$.

8. SRIVASTAVA, K.N. and LOWENGRUB, M. Finite Hilbert transform techniue for triple integral equations with trignometric kernels. Proc. R. Soc. Ednib. 39(1970), 309-321.

9. SHAIL, R. A class of singular integral equation with some applications, Int. J. Math. Educ. Techno1., 15(1984), 359-374.

10. CARLEMAN, T. Uber die Abelsche Integralgleichung mit konstanten Integrationsgrezen, Math. Z., 15(1911), 11-120.

11. COOKE, J.C. The solution of some integral equations and their connection with dual integral equations and series, Glasgow Math. J., 11(1970) 9-20.

12. WI LLIAMS, W.E. A note on integral equations, Glasgow Math. J., 13(1972), 119-121.

13. LAL, BANSI and JAIN, D.L. Uniform streaming past a fixed semi-circular infinite strip, J. Math. Phy. Sci. 17(1978), 365-376.

14. DUTTA, M., and DEBNATH, L. Elements of the Theory of El1iptic and Associated Functions with Applications, Wor1d Press, Calcutta (1965). 


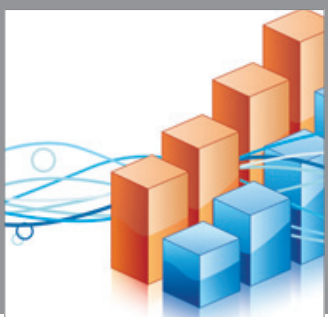

Advances in

Operations Research

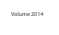

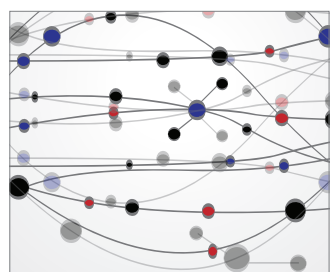

\section{The Scientific} World Journal
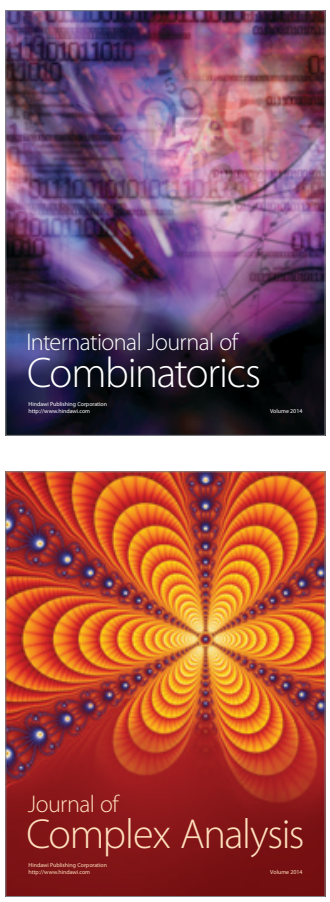

International Journal of

Mathematics and

Mathematical

Sciences
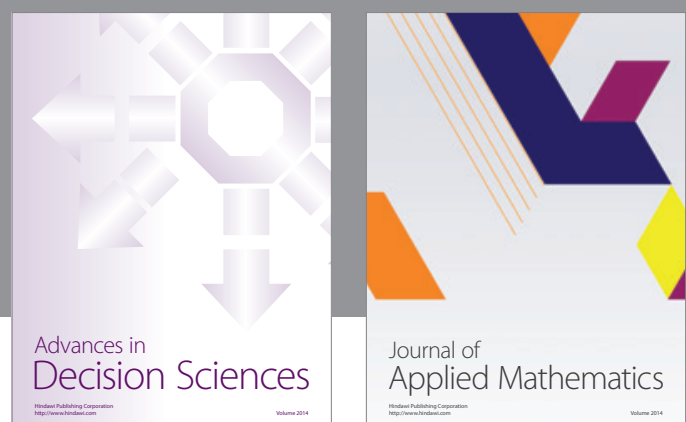

Journal of

Applied Mathematics
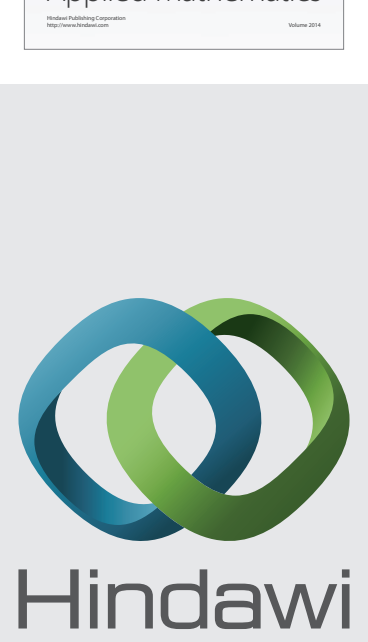

Submit your manuscripts at http://www.hindawi.com
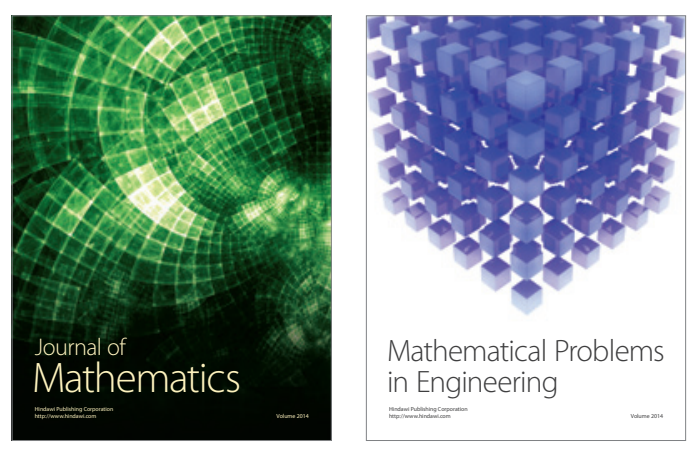

Mathematical Problems in Engineering
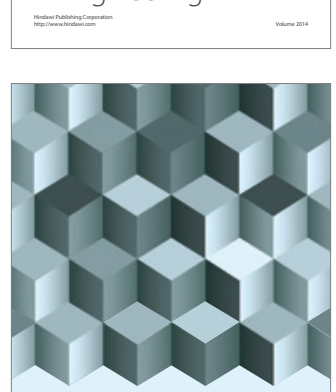

Journal of

Function Spaces
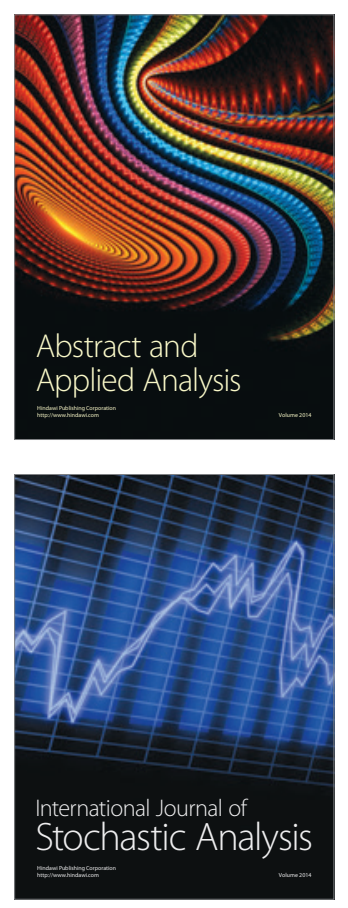

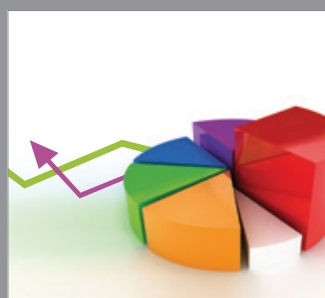

ournal of

Probability and Statistics

Promensencen
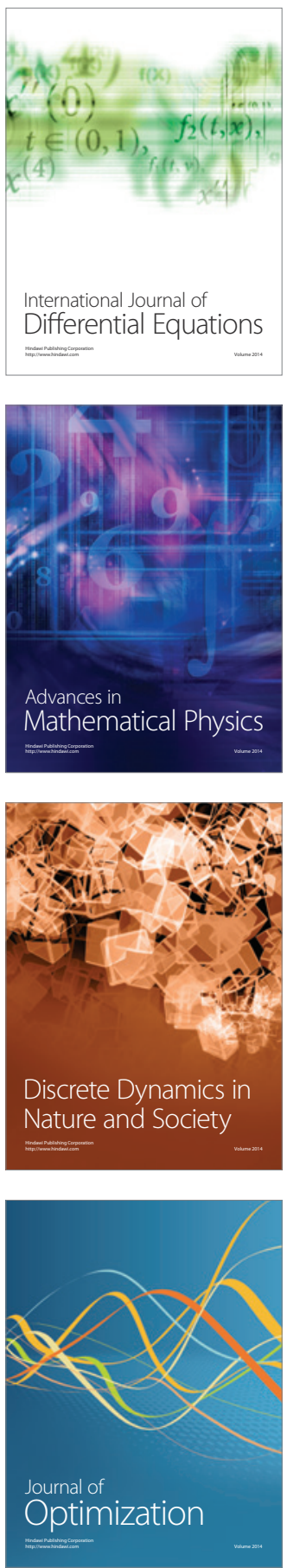